\title{
Organ Culture
}

National Cancer Institute

\section{Source}

National Cancer Institute. Organ Culture. NCI Thesaurus. Code C16941.

The maintenance or growth of tissues, organ primordia, or the parts or whole of an organ in vitro in such a way as to allow differentiation or preservation of the architecture or function. 